brane bound and soluble forms. Cell 1990 $63: 235-43$.

5. Zsebo KM, Wypych J, McNiece IK, et al. Identification, purification, and biological characterization of hematopoietic stem cell factor from buffalo rat liver-conditioned medium. Cell $1990 ; 63: 195-201$.

6. Martin F H, Suggs SV, Langley KE, et al. Primary structure and functional expression of rat and human stem cell factor DNAs. Cell 1990 ; 63 : 203-11.

7. Zsebo KM, Williams DA, Geissler EN, et al. Stem cell factor is encoded at the SI locus of the mouse and is the ligand for the c-kit tyrosine kinase receptor. Cell 1990 ; 63 : 213-24.

8. Huang E, Nocka K, Beier DR, et al. The hematopoietic growth factor $\mathrm{KL}$ is encoded by the SI locus and is the ligand of the c-kit receptor, the gene product of the $\mathrm{W}$ locus. Cell $1990 ; 63: 225-33$.

9. Flanagan JG, Leder P. The kit ligand : a cell surface molecule altered in steel mutant fibroblasts. Cell $1990 ; 63: 185-94$.

10. Matsui Y, Zsebo KM, Hogan BL. Embryonic expression of a haematopoietic growth factor encoded by the $S I$ locus and the ligand for c-kit. Nature 1990 ; 347 : 667-8.

\section{口II BRÈVE $\square \square \square$}

Le facteur natriurétique atrial stimule in vitro la sécrétion d'érythropoiétine. L'hypoxie stimule la production de facteur natriurétique atrial (ANF) et également celle d'érythropoïétine (Epo). Ueno et al. [1], New Orleans (Louisiane, USA), ont étudié l'effet de l'ANF sur des cellules de carcinome rénal humain, maintenues en culture, produisant de l'Epo. Ces cellules ont des sites de liaison de haute affinité pour l'ANF. L'incubation de ces cellules avec des doses croissantes d'ANF entraîne une nette stimulation de la sécrétion d'Epo. L'action de l'ANF passe par la stimulation de la synthèse intracellulaire de GMP cyclique. Dans cette étude, l'ANF, en présence d'un inhibiteur de la phosphodiestérase, augmente les taux intracellulaires de GMP cyclique. Le GMP cyclique pourrait ainsi être le médiatcur responsable de l'effet de l'ANF sur la production d'Epo. Cependant Ueno et al. n'ont pas pu démontrer jusqu'à présent un effet comparable sur des ccllules rénales normales en culture.

[1. Ucno M, et al. Am J Physiol 1990 ; 259 : C427-31.]

$\mathrm{m} / \mathrm{s} n^{\circ} 10$, vol. 6 , décembre 90

\title{
Un nouveau membre de la superfamille des récepteurs nucléaires activé par les inducteurs peroxysomiaux
}

Les inducteurs peroxysomiaux sont, pour certains, utilisés en thérapeutique humaine comme hypolipémiants. Ils induisent, en effet, une importante prolifération des peroxysomes hépatiques, associée à une stimulation de la $\beta$-oxydation des acides gras à longue chaîne par augmentation de la synthèse des enzymes impliquées dans cette voie métabolique. Leur inocuité a été plusieurs fois mise en doute car ils sont, chez les rongeurs, hépatocarcinogènes. Ils représentent d'ailleurs une classe particulière de carcinogènes car ils n'ont aucun pouvoir mutagène détectable, contrairement à la grande majorité des carcinogènes "génotoxiques". Le mécanisme de leur pouvoir tumorigène pourrait être l'hyperproduction d' $\mathrm{H}_{2} \mathrm{O}_{2}$ liée à l'hyperplasie peroxysomiale, eau oxygénée engendrant des radicaux libres oxydants pouvant léser, indirectement, l'ADN.

Une protéine intracellulaire liant ce type de molécule a été décrite, ce qui, joint au pouvoir activateur de ces composés sur l'expression de nombreux gènes, a fait proposer l'hypothèse selon laquelle ils agiraient par l'intermédiaire de récepteurs nucléaires de la même famille que ceux des hormones stéroïdes, thyroïdiennes et de l'acide rétinoïque. I. Issemann et S. Green, en GrandeBretagne, ont voulu tester cette hypothèse en examinant des clones d'ADNc hépatiques codant pour des récepteurs de cette famille mais ne correspondant à aucun de ceux décrits jusqu'à présent [1]. De fait, ils ont trouvé une séquence codant pour un PPAR (peroxisome proliferatoractivated receptor) dont les propriétés sont cohérentes avec celles attendues pour un médiateur de l'action des inducteurs peroxysomiaux. Un ADNc hybride a été synthétisé par génie génétique, codant pour le domaine de liaison à l'ADN des récepteưrs des glucocorticoïdes ou des œstrogènes et la région supposée de liaison du ligand du PPAR. Le vecteur d'expression permettant la synthèse de ce récepteur chimérique a été introduit par transfection dans des cellules, en même temps qu'un gène test contrôlé par un élément de réponse aux glucocorticoïdes ou aux œstrogènes. Cette stratégie, d'utilisation très générale lorsqu'il s'agit de tester la spécificité de ligand d'un récepteur nucléaire dont on ne connaît pas la cible sur l'ADN, est fondée sur le caractère modulaire de ces protéines récepteurs. Un domaine de liaison d'un ligand $\mathrm{A}$ associé à une région de fixation sur une cible $B$ connue d'ADN (ici des éléments de réponse à des hormones stéroïdes) doit permettre au ligand A testé d'activer un gène contrôlé par l'élément B. Ainsi, dans l'expérience d'Issemann et Green, le traitement des cellules transfectées stimule-t-il de 10 à 40 fois l'expression du gène test. Néanmoins, la molécule PPAR ne semble pas avoir d'affinité pour l'inducteur peroxysomial testé, la nafénopine, ce qui suggère qu'elle est différente de la protéine de liaison préalablement décrite. L'effet pourrait donc être indirect, que la nafénopine induise la libération d'un ligand ou une activation par modification du récepteur, par exemple une phosphorylation. Quoi qu'il en šioit, ces résultats montrent que les membres de la superfamille des récepteurs nucléaires tiennent sous leur contrôle une grande diversité de phénomènes, ici impliqués dans le métabolisme lipidique et, peut-être, certaines formes de carcinogenèse hépatique.

A. $\mathbf{K}$.

1. Issemann I, Green S. Activation of a mem ber of the steroid hormone receptor superfamily by peroxisome proliferators. Nature $1990 ; 347: 645-50$ 